\title{
Moderate effect of GGCX polymorphisms on patients warfarin dosage requirement- a meta-analysis
}

\author{
Zhiying Luo ${ }^{1}$, Xi Li ${ }^{1}$, Mouze Liu ${ }^{1}$, Yan Shu ${ }^{1}$, Jiye Yin ${ }^{1}$, Xiaoping Chen ${ }^{1}$, Jianquan Luo ${ }^{1}$, \\ Xiaobing $\mathrm{Li}^{2}$, Wei Zhang, ${ }^{1, *}$ \\ ${ }^{1}$ Department of Clinical Pharmacology, Xiangya Hospital, Central South University, Changsha 410008, P. R. China, Institute of Clinical \\ Pharmacology, Central South University, Hunan Key Laboratory of Pharmacogenetics, Changsha 410078, P. R. China \\ ${ }^{2}$ Department of Cardio-Thoracic Surgery, the Second Xiangya Hospital Hospital of Central South University, Middle Ren-Min Road No. 139, \\ Changsha, Hunan 410011, PR China
}

Email address:

yjsd2003@163.com (Wei Zhang),Dale75@126.com (Xiaobing Li)

\section{To cite this article:}

Zhiying Luo, Xi Li, Mouze Liu, Yan Shu, Jiye Yin, Xiaoping Chen, Jianquan Luo, Xiaobing Li, Wei Zhang. Moderate Effect of GGCX Polymorphisms on Patients Warfarin Dosage Requirement- A Meta-Analysis. American Journal of Life Sciences. Special Issue: Pharmacogenomics \& Personalized Medicine. Vol. 3, No. 1-4, 2015, pp. 7-13. doi: 10.11648/j.ajls.s.2015030104.12

\begin{abstract}
Objective: Association studies on the effects of $G G C X$ gene polymorphisms on warfarin stable dose have shown conflicting results. The aim of this study is to quantitatively summarize whether $G G C X$ gene polymorphisms have potential roles in warfarin dose requirement. Methods: Publications were searched in PubMed, Medline and ISI Web of Knowledge and chosen by exact inclusion and exclusion criteria. A meta-analysis was conducted by using Revman 5.0 software to determine the association between common polymorphisms of the three genes and warfarin dose requirement. Results: Data were extracted from 13 publications with 4167 patients enrolled. Two common polymorphisms (rs699664, rs12714145) of GGCX were included for further meta-analyses. Comparing to rs699664AA geontype carriers, rs699664GG genotype carriers requered higer $3 \%$ [95\% CI: $2 \%-4 \%$, P-valus $<0.0001]$ warfarin dose. The warfarin dosage requirement showed no significant difference between rs699664GG and rs699664GA genotype carriers, $\mathrm{P}=0.51$. Compared to rs12714145AA carriers, the GG and GA genotype carriers needed 5\% (95\% CI, 1\% - 9\%; P = 0.01) and 4\% (95\% CI, 1\% - 8\%; P = 0.02) lower warfarin dosage, respectively. The warfarin dosage requirement showed no significant difference between GG and GA genotype carriers, $\mathrm{P}=0.12$. Conclusion: Our study showed that $G G C X$ polymorphisms were significantly associated with warfarin dose requirement. These polymorphisms should be considered in future warfarin personalized treatment.
\end{abstract}

Keywords: $G G C X$, Warfarin, Polymorphism, Meta-Analysis

\section{Introduction}

Warfarin is the most widely prescribed oral anticoagulant for reducing thromboembolic events in patients with atrial fibrillation, heart valve replacement, deep vein thrombosis and pulmonary embolism ${ }^{1-3}$. However, the existing problems in warfarin clinical application including large individual and ethnic variability in the anticoagulant effect, narrow therapeutic index and serious adverse effects and so on ${ }^{4}$. In the clinical practice, warfarin dosage needs to be carefully adjusted based on international normalization ratio (INR) and must to be maintained within a therapeutic range ${ }^{5}$.

Previous studies have identified many clinical, genetic and environmental factors have different effects on the variability of warfarin maintain dose ${ }^{6,7}$. And it has also been widely accepted that the reasnos for warfarin dose discrepancy are mainly due to genetic factors, among which polymorphism $(-1639 \mathrm{G}<\mathrm{A})$ in the drug target vitamin $\mathrm{K}$ epoxide reductase complex subunit 1 gene (VKORC1) and polymorphisms (R144C, I359L) of the main warfarin metabolizing gene cytochrome-P450 2C9 (CYP2C9) can explain the most part ${ }^{8,9}$.

The anticoagulant mechanism of warfarin is by reducing the regeneration of vitamin $\mathrm{K}$ hydroquinone from vitamin $\mathrm{K}$ epoxide in the vitamin $\mathrm{K}$ cycle in the liver. ${ }^{10}$. Post-translational modification of glutamate to gamma carboxyl glutamate is required for the activity of vitamin $\mathrm{K}$-dependent proteins, like factors II, VII, IX, X and protein C, $\mathrm{S}$ and $\mathrm{Z}$ which are all involved in the coagulation pathway. And the carboxylation effect of these proteins is accomplished by the enzyme gamma-glutamyl carboxylation $(G G C X)$, 
which was located in the rough endoplasmic reticulum and Golgi apparatus ${ }^{11}$. It suggested that GGCX paly a key role in Vitamin K cycle.

To data, studies on the association of GGCX polymophisms with warfarin dosage have been widely explored. However, the results still remian inconclusive and conflicting. The aim of this study is to provide a comprehensive assessment on the association between $G G C X$ polymorphisms and WSD. We collected all available publications on pharmacogenetic studies of the inference of these polymorphisms to warfarin stable dose, and given a quantitatively study using meta-analysis.

\section{Materials and Methods}

\subsection{Search Strategy}

All clinical studies on $G G C X$ polymorphisms and warfarin stable dose were identified through systematic searches in PubMed and EMBASE published up to Feb 28, 2014. The search terms were " $G G C X$ " or "gamma-glutamyl carboxylase" in combination with warfarin and "polymorphism" or "genotype" or "mutation". The reference lists of reviews and retrieved articles were hand searched at the same time.

\subsection{Inclusion and Exclusion Criteria}

A study was included in the meta-analysis if it was satisfied with following criteria: (1) prospective and retrospective cohort studies, case-control studies and randomized controlled trials were included; (2) genotyping of at least one polymorphism of GGCX, (3) sample size and warfarin maintance dose for each genotypes were displayed. If a study did not supply number or ethnicities of patients, mean maintance dose of warfarin, authors were contacted for additional information;

Studies were excluded if any of the following applied: (1) review articles or case report; (2) The inclusion and exclusion criteria for study patients were undefined; (3) papers written in a language other than English or Chinese;

\subsection{Data Extraction}

A double-blinded search and identification of eligible articles based on the above inclusion criteria were carried out independently by two researchers (Zhi-Ying Luo and Xiao-Bing Li). The data recording form contains the first author's name, year of publication, ethnicity (country), number of patents, sex, age (mean and SD), genotype method, target INR range, indication of warfarin and warfarin dose of each genotype. After exacted data were reviewed and compared by a third reviewer (Wei Zhang), and the discrepancies between extractors were discussed and solved with consensus.

If the study provide medians and interquartile ranegs or medians and ranges (minimum - maximum) instead of means and SDs, we imputed the means ans SDs as described in Cochrane handbooke and Hozo et al. ${ }^{12}$.

\subsection{Statistical Methods}

The units of warfarin dosage were differently represented as dose/week by some authors and as dose/day by others. In order to uniform the warfarin dosage before meta-analyses, we divided the mean dose and associated SD in each genotype group by the mean stable dose in the reference groups ${ }^{13-15}$. The warfarin dosage of rs699664GG and rs $12714145 \mathrm{GG}$ genotypes were used as the reference, respectively.

The STATA 12.0 software (StataCorp, College Station, TX, USA) and Revman 5.0 (Cochrane Collaboration) were applied to analyze the relationships between the polymorphisms and warfarin stable dose. The weight of each study is the inverse of its SD of normalized warfarin dose, and the influence of each genotype on warfarin dose requirement was expressed as mean difference (MD). After normalizated, the calculated MDs representen relative differences rather than absolute differences in stable dose. For example, a mean difference of 0.1 would indicate a $10 \%$ increase in warfarin dose requirement. The indicator of effect used weight mean difference (WMD), and sum of each WMD equal to total WMD. The impact of gene polymorphisms on warfarin stable dose was examined by means of $\mathrm{Z}$ test, and the significant level was set as a $P$ value less than 0.05 .

The heterogeneity of publications in each meta-analysis was evaluated by Mantel-Haenszel chi-squared test (Cochran's $Q$ test) and calculation of the variation across studies attributable to heterogeneity. If the Cochran $\mathrm{Q}$ test $P$ value $<0.1$, the random-effect model was chosen to calculate the overall MD, otherwise a fix-effect model was used. To further evaluate the extent of heterogeneity between publications, $\left(\mathrm{I}^{2}\right)$ test was employed, and the values of $\left(\mathrm{I}^{2}\right)$ different levels $(25 \%, 50 \%, 75 \%)$ were considered as low, moderate and high heterogeneity, respectively.

In order to eliminate the sources of heterogeneity, we conducted the sensitive analysis by deselecting studies one by one in chronological order. We conducted this procedure by removing one study and the rest were analyzed to evaluate whether the results were affected statistically significantly. Publication bias was examined by means of Funnel Plot where normalized dose requirement were plotted versus inverse standard error. To further evaluate publication bias, Begg's and Egger's test were also used in this study.

\section{Results}

\subsection{Studies Identification and Characteristics}

We identified 56 published studies with full-text articles examined the relationship between GGCX polymorphisms and warfarin dosage. Of these, only 14 studies were included in the meta-analysis. Maong the included studies, ten of which investigated the effect of GGCX rs699664 polymorphism on warfarin dosage were included, and four studies about the effect of rs 12714145 on warfarin dose were inclueded. A flow chart summarizing the process of study inclusion/exclusion was depicted in Figure 1. A summary of the all included studies was given in Table 1 . A total of 4167 patients were 
included in the meta-analysis.

\subsection{Meta-Analysis}

\subsubsection{Impact of GGCX Gene rs699664 on Warfarin Dosage}

The influence of GGCX rs699664 on warfarin dosage requirement was shown in figure 2 . The total number of patients carring GG, GA and AA genotypes were 1084, 1078 and 298 respectively. Comparing to rs699664AA geontype carriers, rs699664GG genotype carriers requered higer 3\% [95\% CI: $2 \%-4 \%$, P-valus $<0.0001]$ warfarin dose. The warfarin dosage requirement showed no significant difference between rs699664GG and rs699664GA genotype carriers, $\mathrm{P}=0.51$. The analysis of GG vs AA shows homogeneity $(\mathrm{P}=$ $0.09, \mathrm{I}^{2}=40 \%$ ), so fixed-effect model was used. Random-effect mode was chosen in this analysis of GG vs GA because of the statistic heterogeneity $\left(\mathrm{P}=0.00, \mathrm{I}^{2}=83 \%\right)$.

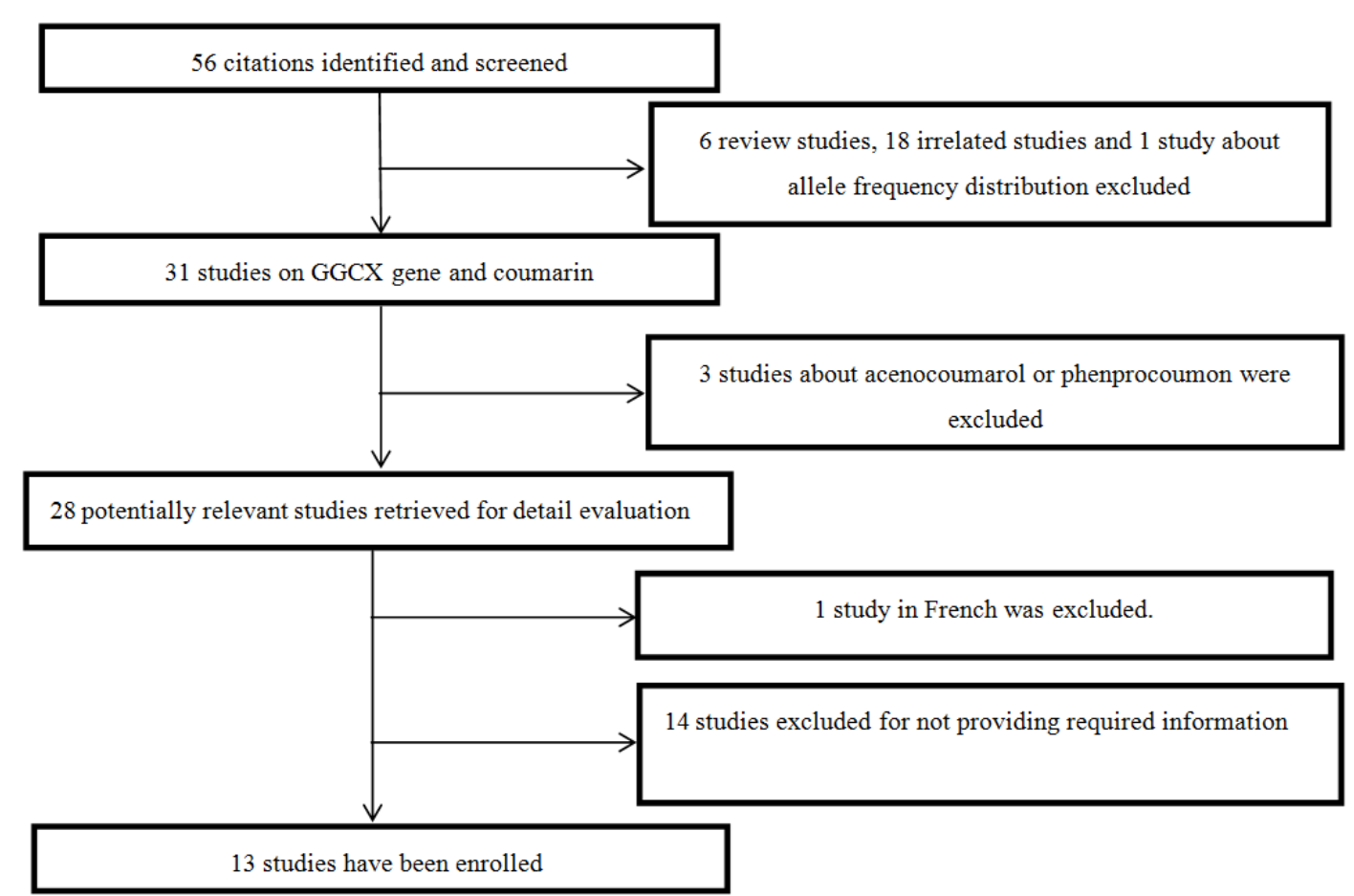

Figure 1. Flow chart of screening excluded studies and its reasons, identified the studies in final analysis.

Table 1. Characteristics of included studies.

\begin{tabular}{|c|c|c|c|c|c|c|c|c|c|c|c|}
\hline \multirow{3}{*}{ Studies } & \multirow{3}{*}{$\begin{array}{l}\text { Study location } \\
\text { or population }\end{array}$} & \multirow{3}{*}{ Number } & \multirow{3}{*}{ Age } & \multirow{3}{*}{$\begin{array}{l}\text { INR target } \\
\text { range }\end{array}$} & \multirow{3}{*}{$\begin{array}{l}\text { Indication of } \\
\text { warfarin }\end{array}$} & \multicolumn{6}{|c|}{ GGCX genotype frequencies } \\
\hline & & & & & & \multicolumn{3}{|c|}{ rs12714145 $(\mathrm{N})$} & \multicolumn{3}{|c|}{ rs699664 (N) } \\
\hline & & & & & & GG & GA & $\mathbf{A A}$ & GG & GA & $\mathbf{A A}$ \\
\hline Shen-Wen Huang, $2011^{16}$ & Chinese (Asian) & $217(90 / 127)$ & $58 \pm 16$ & $1.8-3.0$ & VTD & 84 & 117 & 16 & - & - & - \\
\hline Larisa $H, 2012^{17}$ & $\begin{array}{l}\text { African } \\
\text { Americans }\end{array}$ & $338(98 / 240)$ & $58 \pm 16$ & NR & NR & 71 & 107 & 33 & 46 & 92 & 70 \\
\hline Kyung-Eun Lee, $2012^{18}$ & Korean (Asian) & 191(63/128) & $57.8 \pm 10.1$ & $2.0-3.0$ & HVR & - & - & - & 91 & 77 & 23 \\
\hline Jung Ran Choi, $2011^{19}$ & Korean (Asian) & $564(306 / 258)$ & $63.2 \pm 11.7$ & $2.0-3.0$ & $\begin{array}{l}\text { AF, HVR, PE, } \\
\text { DVT }\end{array}$ & - & - & - & 265 & 250 & 49 \\
\hline Cristi R. King, $2010^{20}$ & Caucasian & $985(486 / 499)$ & $60 \pm 15$ & $1.5-2.0$ & VTE & - & - & - & - & - & - \\
\hline Hedi Schelleman, $2010^{21}$ & $\begin{array}{l}\text { African } \\
\text { Americans } \\
\text { Caucasian }\end{array}$ & $\begin{array}{l}22 \\
36\end{array}$ & NR & $2.0-3.0$ & VTE & - & - & - & $\begin{array}{l}1 \\
12\end{array}$ & $\begin{array}{l}10 \\
22\end{array}$ & $\begin{array}{l}10 \\
2\end{array}$ \\
\hline Masako Ohno, $2009^{22}$ & Janpan (Asian) & $125(75 / 50)$ & $73.1 \pm 11.6$ & $1.5-3.0$ & AF, DVT, PE & - & - & - & 68 & 47 & 10 \\
\hline Rina Kimura, $2007^{23}$ & Janpan (Asian) & 93 & $68.1 \pm 10.6$ & $1.6-2.6$ & CEI, EI & - & - & - & 48 & 39 & 6 \\
\hline Manuela Vecsler, $2006^{24}$ & Israel & $100(52 / 48)$ & $62(18-88)$ & $1.9-4.2$ & NR & - & - & - & 50 & 41 & 9 \\
\hline Ronen Loebstein, $2005^{25}$ & Israel & $100(52 / 48)$ & $62(18-88)$ & $1.9-4.2$ & NR & - & - & - & 50 & 41 & 9 \\
\hline Huang SW, $2011^{26}$ & Chinese (Asian) & $249(111 / 138)$ & $51.4 \pm 14.9$ & $1.8-3.0$ & $\mathrm{AF}$ & 97 & 134 & 18 & - & - & - \\
\hline Yundan Liang, $2013^{27}$ & Chinese (Asian) & $300(138 / 162)$ & $47.9 \pm 12.5$ & $1.5-3.0$ & $\begin{array}{l}\text { HVR, DVT, } \\
\text { CABG }\end{array}$ & 104 & 146 & 33 & 119 & 137 & 24 \\
\hline Shi-Long Zhong, $2011^{28}$ & Chinese (Asian) & $845(478 / 367)$ & $\begin{array}{l}47.9(38.9- \\
55.8)\end{array}$ & $1.8-3.0$ & HVR & - & - & - & 383 & 383 & 75 \\
\hline
\end{tabular}

Note: DVT =Deep Vein Thrombosis, NR =Not Referred, HVR =Heart Valve Replacement, AF =Atrial Fibrillation, VTE =Venous Thromboembolism, $\mathrm{CABG}=$ Coronary Artery Bypass Graft, $\mathrm{PE}=$ Pulmonary Embolism, MI: myocardial infarction, ASO, arteriosclerosis obliterans. AV: artificial vessel, CEI $=$ Cardioembolic infarction, $\mathrm{EI}=$ Embolic infraction 


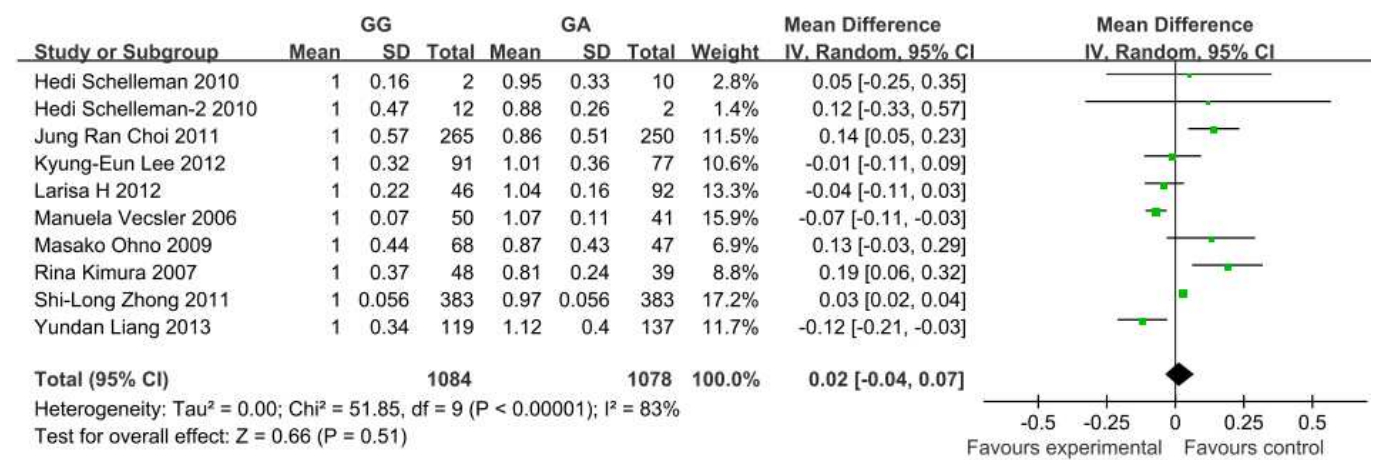

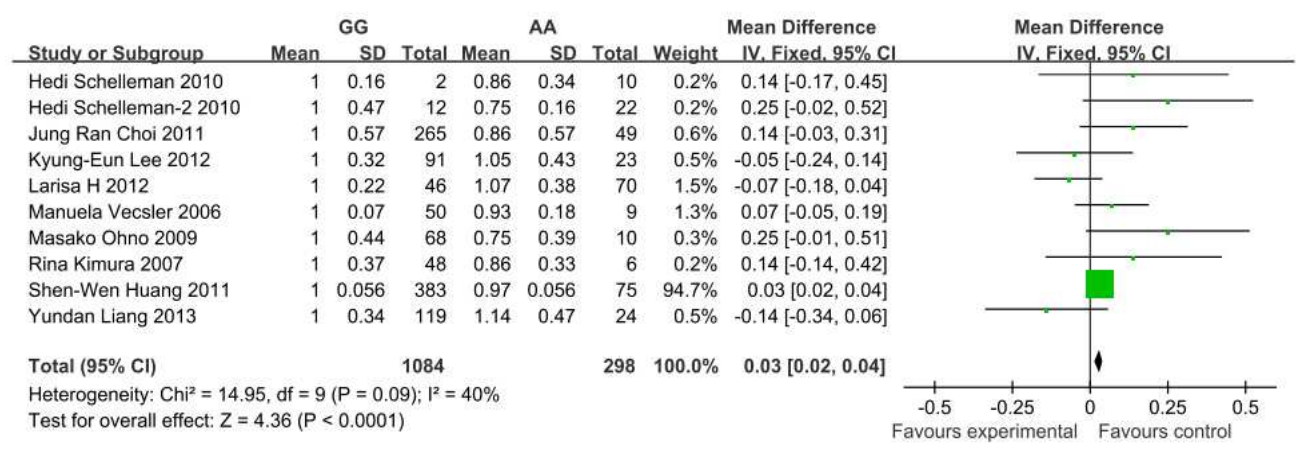

Figure 2. Forest plot of the impact of GGCX rs699664 polymorphism on warfarin dose requirement.

\subsubsection{Impact of GGCX rs12714145 Polymorphism on Warfarin Dosage Requirement}

The impact of GGCX rs12714145 polymorphism on warfarin dose requirement was described in Fig 3. Only 4 publications were included in our study with pooled data of 649 patients (356 GG, 405 GA and 100 AA respectively). No statistic heterogeneities were found through meta-analyses ( $P$
$=0.42, \mathrm{I}^{2}=0 \%$ for GG $v s . \mathrm{GA} ; \mathrm{P}=0.17, \mathrm{I}^{2}=40 \%$ for $\mathrm{GG} v s$. AA, $\mathrm{P}=0.45, \mathrm{I}^{2}=0 \%$ for GA $v$ s AA), so the fixed-effect model was chosen. Compared to GG and GA genotype carriers, AA carriers needed 5\% (95\% CI, 1\% - 9\%; P = 0.01) and 4\% (95\% CI, $1 \%-9 \% ; \mathrm{P}=0.01$ ) higher warfarin dosage. The warfarin dosage requirement showed no significant difference between GG and GA genotype carriers, $\mathrm{P}=0.12$.

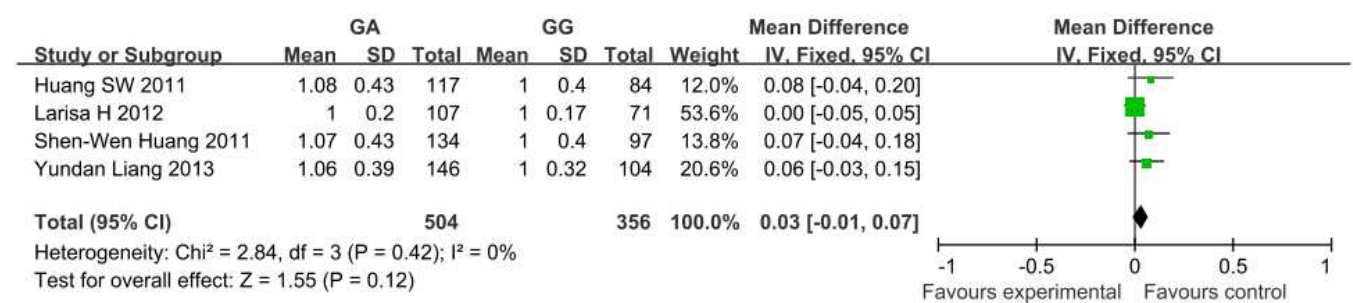

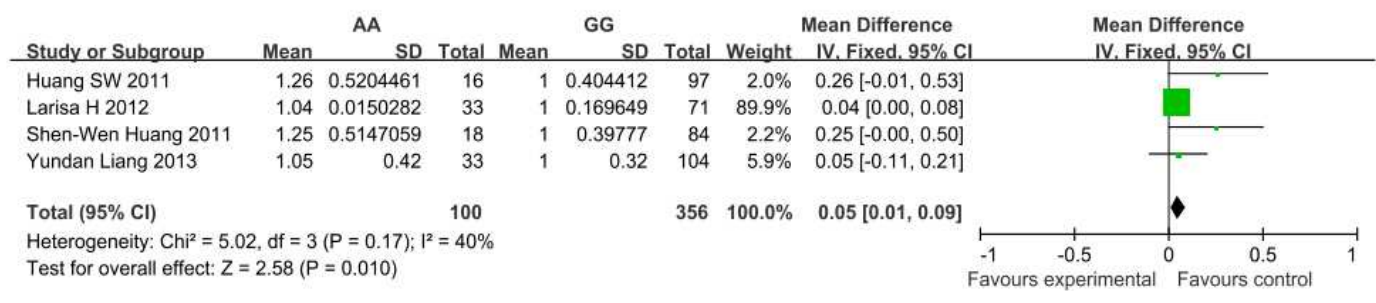

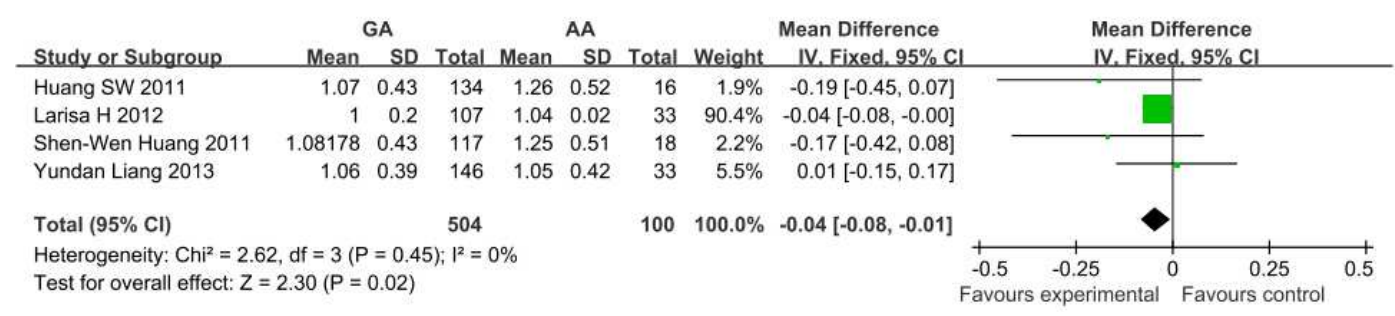

Figure 3. Forest plot of the impact of GGCX rs 12714145 polymorphism on warfarin dose requirement. 


\subsubsection{Sensitive Analysis and Publication Bias}

All meta-analyses about GGCX rs699664 polymorphsim and warfarin dose requirement showed evidence of statistical heterogeneity, and a random-effects model was used in these meta-analyses. We first conducted the sensitive analyses by deselecting studies one by one in chronological order, and found no significant difference or revisal change between these studies and original outcomes.

We also conducted subgroup analyses according to population diversities if statistic heterogeneity was found. For all of these meta-analyses conducted in this study, only the compare between GGCX rs699664GG and GA genotypes showed significant statistic heterogeneity. Of the 10 included studies about the relationship between rs699664GG and GA polymorphism and warfarin dose requirement, only one study presented the effect in Caucasian population, so the subgroup analysis was not carried out in this category. More than two studies had been performed in each subgroup. The warfarin dosage showed no significant difference between rs699664GG and rs699664GA genotype carriers eithor in African-Amercian or Asian population, like shown in Table 2. Because of statistic heterogeneity in the Asian population group, a further subgroup was conducted in these studies according to country diversities. And resultes showed that GGCX rs699664 GG genoypes need higher 0.17 (95\% CI: $0.07-0.27, \mathrm{P}=0.01$ ) warfarin than GA genotypes.

Funnel plots of each analysis did not indicate any asymmetries suggestive of publication bias (data not shown).

Table 2. Warfarin dosage requirement between GGCX rs699664 GG vs GA genotypes in different populations.

\begin{tabular}{llllll}
\hline \multirow{2}{*}{ Subgroups } & Number & Heterogeneity & & Mean difference (95\% CI) & P \\
\cline { 3 - 4 } & $\mathbf{P}$ & $\mathbf{I}^{\mathbf{2}}$ & & \\
\hline Ethicity & 2 & 0.32 & $0 \%$ & $-0.04(-0.01,0.03)$ & 0.32 \\
African-Amercian & 7 & 0.00 & $88 \%$ & $0.03(-0.04,0.09)$ & 0.42 \\
Asian & 2 & 0.04 & $77 \%$ & $0.07(-0.08,0.21)$ & 0.37 \\
Country & 2 & 0.57 & $0 \%$ & $0.17(0.07,0.27)$ & 0.01 \\
Chinese & 2 & 0.00 & $90 \%$ & $-0.04(-0.18,0.11)$ & 0.61 \\
Janpanese & & & \\
Korean & &
\end{tabular}

\section{Discussion}

The aim of this meta-analysis was to identify wthether GGCX polymorphisms had a potential effect on warfarin dosage. The resultes of our meta-analysis suggested that GGCX rs699664AA carriers need lower warfarin dosage than wild homozygous(GG) carriers, and GGCX rs12714145AA carriers required higher warfarin dose than GG and GA genotype carriers.

Polymorphim of GGCX gene was first reported associated with rare autosomal recessive bleeding disorders by Brenner B et al. in $1998^{29}$, and the further animal experiment further provied clear proof of the key role of GGCX for the functon of these Vitamin K-dependent protiens ${ }^{30}$. GGCX rs699664 polymorphism was a demonstrated functional mutation, which causes a missense change $\mathrm{R} 325 \mathrm{Q}^{31}$. The resultes of our analyses showed that 699664 GG carriers need higher dose than AA carriers, and the resultes of subgroup analyses demonstrated that the GGCX rs699664GG genotypes had higher warfarin dose requirement than GA geotypes in Janpanese. The were statistic heterogeneities between the analyses in Chinese and Korean population. The data of Shi-Long Zhong et al. study was presented in median and quartiles, and we translated them into mean and SD - and the main reason for its heterogentity in Chinese population. The daily warfain dose of the two studies in Korean population showed significant difference $(\mathrm{P}<0.05)$, and this difference may lead to statistic heterogeneity.

The polymorphism of GGCX rs12714145 (G3261A) located in intron 2 was identified as one predictor of warfarin dose in our study, AA carriers required higher warfarin dose than GG and GA genotype carriers. Wadelius et al. has reported that many GGCX single nucleotide polymorphisms (SNPs) are in high disequilibrium linkage (LD) in Caucasian population ${ }^{32}$. And we hypothsis that other functional SNPs may be in high LD with GGCX (G3261A).

All of the meta-analyses on the two polymorphisms of GGCX with confirmed association with warfarin stable dose showed no study bias, which means the results are reliable. In addition, in the sensitive analyses conducted in our study showed no statistic changes or reversal results by deselecting studies one by one in chronological order, which means the result of present meta-analyses were stable.

The clinical use of warfarin is always a challenge to doctors and researcher. Nevertheless, the era of using one standard fixed dose for all patients is be replaced by personalized warfarin treatment because of breakthroughs in the parmacogenetics studies of warfarin. Even though warfarin dosage inter-individual and inter-ethnicity difference can be mainly explained by VKORC1 and CYP2C9 gene polymorphisms, other factors, including age, gender, body mass index, health conditions, co-medications and so on, also had important influence on warfarin sensitive ${ }^{33}$. Other genes such as summarized in this study that might have important role in warfarin dose variation are being characterized.

The main limitation of our meta-analysis is that the warfarin mean dosages applied to our meta-analysis have not been adjusted for other genetic and non-genetic predictors of dose as described above. The other is that data of some study was presented as figures or not able to acquire the mean warfarin dose.

This is the first meta-analysis about the impact of GGCX 
(rs699664 and rs12714145) gene polymorphisms on warfairn dose requirement. And we found that GGCX polymorphisms had moderate but significant impact on warfarin dose inter-individual difference. Future studies of genes with smaller effects might be the key to improve the prediction accuracy of warfarin pharmacogenetic dosing algorithm. The results of our research reinforce the relationship between GGCX polymorphisms and

\section{Acknowledgments}

This work was supported by the National Scientific Foundation of China (No. 81273595, 30901834, 81001476), the Scientific Foundation of Hunan (No. 11K073, 10JJ4020), the "863" Project (No. 2012AA02A518) and NCET-10-0843. All the authors had complete access to the data and have full responsibility for the decision to submit the report.

\section{References}

[1] Wadelius M, Pirmohamed M. Pharmacogenetics of warfarin: current status and future challenges. Pharmacogenomics J. 2007 2007-04-01;7(2):99-111.

[2] The effect of low-dose warfarin on the risk of stroke in patients with nonrheumatic atrial fibrillation. The Boston Area Anticoagulation Trial for Atrial Fibrillation Investigators. N Engl J Med. 1990 1990-11-29;323(22):1505-11.

[3] Connolly SJ, Laupacis A, Gent M, Roberts RS, Cairns JA, Joyner C. Canadian Atrial Fibrillation Anticoagulation (CAFA) Study. J Am Coll Cardiol. 1991 1991-08-01;18(2):349-55.

[4] Burns M. Management of narrow therapeutic index drugs. J Thromb Thrombolysis. 1999 1999-04-01;7(2):137-43.

[5] Hirsh J, Dalen J, Anderson DR, et al. Oral anticoagulants: mechanism of action, clinical effectiveness, and optimal therapeutic range. Chest. 2001 2001-01-01;119(1 Suppl):8S-21S.

[6] Anderson JL, Horne BD, Stevens SM, et al. Randomized trial of genotype-guided versus standard warfarin dosing in patients initiating oral anticoagulation. Circulation. 2007 2007-11-27;116(22):2563-70.

[7] Klein TE, Altman RB, Eriksson N, et al. Estimation of the warfarin dose with clinical and pharmacogenetic data. N Engl J Med. 2009 2009-02-19;360(8):753-64.

[8] Takahashi H, Wilkinson GR, Nutescu EA, et al. Different contributions of polymorphisms in VKORC1 and CYP2C9 to intra- and inter-population differences in maintenance dose of warfarin in Japanese, Caucasians and African-Americans. Pharmacogenet Genomics. 2006 2006-02-01;16(2):101-10.

[9] Takahashi H, Wilkinson GR, Nutescu EA, et al. Different contributions of polymorphisms in VKORC1 and CYP2C9 to intra- and inter-population differences in maintenance dose of warfarin in Japanese, Caucasians and African-Americans. Pharmacogenet Genomics. 2006 2006-02-01;16(2):101-10.

[10] Moreau C, Loriot MA, Siguret V. [Vitamin K antagonists: from discovery to pharmacogenetics]. Ann Biol Clin (Paris). 2012 2012-10-01;70(5):539-51.
[11] Stafford DW. The vitamin K cycle. J Thromb Haemost. 2005 2005-08-01;3(8):1873-8.

[12] Hozo SP, Djulbegovic B, Hozo I. Estimating the mean and variance from the median, range, and the size of a sample. BMC Med Res Methodol. 2005 2005-01-20;5:13.

[13] Lindh JD, Holm L, Andersson ML, Rane A. Influence of CYP2C9 genotype on warfarin dose requirements--a systematic review and meta-analysis. Eur J Clin Pharmacol. 2009 2009-04-01;65(4):365-75.

[14] Jorgensen AL, FitzGerald RJ, Oyee J, Pirmohamed M, Williamson PR. Influence of CYP2C9 and VKORC1 on patient response to warfarin: a systematic review and meta-analysis. PLoS One. 2012 2012-01-20;7(8):e44064.

[15] Yang L, Ge W, Yu F, Zhu H. Impact of VKORC1 gene polymorphism on interindividual and interethnic warfarin dosage requirement--a systematic review and meta analysis. Thromb Res. 2010 2010-04-01;125(4):e159-66.

[16] Huang SW, Xiang DK, Huang L, et al. Influence of GGCX genotype on warfarin dose requirements in Chinese patients. Thromb Res. 2011 2011-02-01;127(2):131-4.

[17] Cavallari LH, Perera M, Wadelius M, et al. Association of the GGCX (CAA)16/17 repeat polymorphism with higher warfarin dose requirements in African Americans. Pharmacogenet Genomics. 2012 2012-02-01;22(2):152-8.

[18] Lee KE, Chang BC, Kim HO, et al. Effects of CYP4F2 gene polymorphisms on warfarin clearance and sensitivity in Korean patients with mechanical cardiac valves. Ther Drug Monit. 2012 2012-06-01;34(3):275-82.

[19] Choi JR, Kim JO, Kang DR, et al. Proposal of pharmacogenetics-based warfarin dosing algorithm in Korean patients. J Hum Genet. 2011 2011-04-01;56(4):290-5.

[20] King CR, Deych E, Milligan P, et al. Gamma-glutamyl carboxylase and its influence on warfarin dose. Thromb Haemost. 2010 2010-10-01;104(4):750-4.

[21] Schelleman H, Brensinger CM, Chen J, Finkelman BS, Rieder MJ, Kimmel SE. New genetic variant that might improve warfarin dose prediction in African Americans. Br J Clin Pharmacol. 2010 2010-09-01;70(3):393-9.

[22] Ohno M, Yamamoto A, Ono A, et al. Influence of clinical and genetic factors on warfarin dose requirements among Japanese $\begin{array}{lllll}\text { patients. Eur J Clin Pharmacol. } 2009 & \end{array}$ 2009-11-01;65(11):1097-103.

[23] Kimura R, Miyashita K, Kokubo Y, et al. Genotypes of vitamin $\mathrm{K}$ epoxide reductase, gamma-glutamyl carboxylase, and cytochrome P450 2C9 as determinants of daily warfarin dose in Japanese patients. Thromb Res. 2007 2007-01-20;120(2):181-6.

[24] Vecsler M, Loebstein R, Almog S, et al. Combined genetic profiles of components and regulators of the vitamin K-dependent gamma-carboxylation system affect individual sensitivity to warfarin. Thromb Haemost. 2006 2006-02-01;95(2):205-11.

[25] Loebstein R, Vecsler M, Kurnik D, et al. Common genetic variants of microsomal epoxide hydrolase affect warfarin dose requirements beyond the effect of cytochrome P450 2C9. Clin Pharmacol Ther. 2005 2005-05-01;77(5):365-72. 
[26] Huang SW, Xiang DK, Wu HL, Chen BL, An BQ, Li GF. [Impact of five genetic polymorphisms on inter-individual variation in warfarin maintenance dose]. Zhonghua Yi Xue Yi Chuan Xue Za Zhi. 2011 2011-12-01; 28(6):661-5.

[27] Liang Y, Chen Z, Guo G, et al. Association of genetic polymorphisms with warfarin dose requirements in Chinese patients. Genet Test Mol Biomarkers. 2013 2013-12-01; 17(12): 932-6.

[28] Zhong SL, Liu Y, Yu XY, et al. The influence of genetic polymorphisms and interacting drugs on initial response to warfarin in Chinese patients with heart valve replacement. Eur J Clin Pharmacol. 2011 2011-06-01; 67(6):581-90.

[29] Brenner B, Sanchez-Vega B, Wu SM, Lanir N, Stafford DW, Solera J. A missense mutation in gamma-glutamyl carboxylase gene causes combined deficiency of all vitamin K-dependent blood coagulation factors. Blood. 1998 1998-12-15; 92(12): 4554-9.
[30] Zhu A, Sun H, Raymond RJ, et al. Fatal hemorrhage in mice lacking gamma-glutamyl carboxylase. Blood. 2007 2007-06-15; 109(12):5270-5.

[31] Kinoshita H, Nakagawa K, Narusawa K, et al. A functional single nucleotide polymorphism in the vitamin-K-dependent gamma-glutamyl carboxylase gene (Arg325Gln) is associated with bone mineral density in elderly Japanese women. Bone. 2007 2007-02-01; 40(2):451-6.

[32] Wadelius M, Chen LY, Downes K, et al. Common VKORC1 and GGCX polymorphisms associated with warfarin dose. Pharmacogenomics J. 2005 2005-01-20; 5(4):262-70.

[33] Jonas DE, McLeod HL. Genetic and clinical factors relating to warfarin dosing. Trends Pharmacol Sci. 2009 2009-07-01; 30(7): 375-86. 